\title{
Accelerometry: A Feasible Method to Quantify Physical Activity in Ambulatory and Nonambulatory Adolescents with Cerebral Palsy
}

\author{
Jan Willem Gorter, ${ }^{1}$ Stephen G. Noorduyn, ${ }^{1,2}$ Joyce Obeid, ${ }^{3}$ and Brian W. Timmons ${ }^{1,3}$ \\ ${ }^{1}$ CanChild Centre for Childhood Disability Research, McMaster University, 1400 Main Street West, IAHS Building, Room 408, \\ Hamilton, ON, Canada L8S 1C7 \\ ${ }^{2}$ Health Research Methodology Program, McMaster University, Hamilton, ON, Canada L8S 4K1 \\ ${ }^{3}$ Child Health \& Exercise Medicine Program, Department of Pediatrics, McMaster University, 1280 Main Street West, \\ HSC 3N27G, Hamilton, ON, Canada L8S 4K1 \\ Correspondence should be addressed to Jan Willem Gorter, gorter@mcmaster.ca
}

Received 29 December 2011; Accepted 23 April 2012

Academic Editor: Roslyn Boyd

Copyright ( $\odot 2012$ Jan Willem Gorter et al. This is an open access article distributed under the Creative Commons Attribution License, which permits unrestricted use, distribution, and reproduction in any medium, provided the original work is properly cited.

Objective. To determine the feasibility of physical activity monitoring in adolescents with cerebral palsy (CP). Methods. A convenience sample of ambulatory and non-ambulatory adolescents $(N=23 ; 17$ males, 6 females; mean age $13.5 \mathrm{y}$, SD $2.6 \mathrm{y}$; Gross Motor Function Classification System (GMFCS) distribution: $n=9$ Level I, $n=5$ Level II, $n=5$ Level III, $n=4$ Level IV) was recruited. Physical activity (PA) was objectively assessed using the ActiGraph GT1M activity monitor. Discomfort or adverse effects of wearing the accelerometers were recorded by participants. Levels of physical activity were determined as total PA, light PA (LPA), moderate PA (MPA), moderate-to-vigorous (MVPA), and vigorous PA (VPA) using cut-points recently validated for CP. Results. Most participants showed little reluctance. Mean daily MVPA for all participants was 30.7 minutes (SD 30.3), which corresponded to 2.7 (SD 2.4) minutes of MVPA per hour or 4.5\% (SD 3.9) of the total monitoring time. Total PA and MVPA were greatest in ambulatory youth (GMFCS levels I and II) compared with youth who use a walking aid or wheelchair (GMFCS levels III and IV) $(P<0.05)$. Conclusion(s). The results support the use of the accelerometer as a feasible and useful measure of activity in ambulatory and nonambulatory adolescents with CP.

\section{Introduction}

Impaired motor function is the hallmark of cerebral palsy (CP). As a result, children and adolescents with CP are at particular risk for inactivity and the associated negative health impacts (e.g., obesity with exacerbated cardiovascular dysfunction) [1-3]. Not surprisingly, physical activity for children and adolescents with CP is commonly prescribed in clinical practice [4]. However, recent intervention studies have not been able to show a sustained increased activity level in the home, school, or community settings $[5,6]$.

We are currently designing the Stay-FIT intervention study to implement and evaluate a community-delivered physical activity programme for youth with $\mathrm{CP}$ across the severity spectrum [7]. This intervention study is part of a translational research programme focusing on physical activity in individuals with CP (http://www.canchild.ca/en/ ourresearch/stay_fit.asp). The Stay-FIT programme will examine issues of physical activity covering several domains described by the World Health Organization's International Classification of Functioning (ICF) [8], ranging from cardiovascular function and structure [9] to performance in physical activity. This programme reflects the recent paradigm shift in therapy for youth with CP by using physical activity as both the focus of intervention and the primary outcome.

In order to design these future interventions, an acceptable outcome measure for physical activity is needed. Accelerometer-based activity monitoring provides an excellent measure of daily physical activity as it can be used to measure the amount, intensity, and pattern of both activity 
and sedentary behavior [10-13]. In 2010, Capio et al. published a validation study on the use of a uniaxial accelerometer (MTI) to monitor activity in 31 ambulatory children with CP, Gross Motor Function Classification System (GMFCS) levels I-III [11]. Although this study did not examine the feasibility of an accelerometer systematically, the authors reported that the participants did not manifest any indications of intolerance with the device. The accelerometer was able to capture raw activity volume in unstructured free play and in six structured activities of increasing intensities including sitting, walking, and jogging. The validity of this device as a measure of PA volume was confirmed by its linear association with measured heart rate and observed PA. GMFCS levels, however, explained 0 and less than $1 \%$ of the variance in activity during structured and free play activities, respectively. It must be noted that the participants' accelerometer data demonstrated a large degree of variance, as shown by the high standard deviations. Therefore, the authors deemed it inappropriate to use regression equations to predict an activity cut-point for MVPA. In 2011, a study using the ActiGraph 7164 accelerometer was published by Clanchy et al. including mainly ambulatory children and adolescents (mean age $12.6 \pm 2.0$ years) classified at GMFCS level I or II with only a small number of GMFCS III subjects [12]. This study showed that the ActiGraph 7164 is able to differentiate between different intensities of walking in children and adolescents with CP. The validity of the ActiGraph accelerometer as a measure of PA was confirmed by using directly measured oxygen uptake as a criterion measure. Unfortunately the data did not provide sufficient power to perform meaningful subgroup analyses by GMFCS level. Van den Berg-Emons et al. reported in 2011 on the physical activity measurement in adults with various diagnoses [10]. In this study a small number of nonambulatory adults with CP were included $(n=4)$ with limited wear time of 2 consecutive days ( 48 hours). The authors were able to show that activity volume and intensity could be measured in nonambulatory adults with $\mathrm{CP}$ during various activities including wheelchair driving. To our knowledge, there have been no studies to date that have evaluated the psychometric properties of accelerometer-based assessments of habitual physical activity in non-ambulant adolescents with $\mathrm{CP}$ (GMFCS level IV and V) [13].

Despite the growing interest in physical activity and the use of accelerometers in the $\mathrm{CP}$ population, there remains a gap in our knowledge on assessment of habitual physical activity at home, at school, and in the community in adolescents with CP, particularly for those with more severe functional limitations [11-13]. In this paper we present the results of the Stay-FIT pilot study that was developed to test the feasibility of accelerometry for use in ambulatory and nonambulatory adolescents with $\mathrm{CP}$.

\section{Materials and Methods}

2.1. Participants. Adolescents were recruited through regional spasticity and teenager-transition clinics of a university medical center. Participants met the following inclusion criteria: (1) age between 10 and 20 years; (2) a definite diagnosis of CP; (3) a GMFCS level I, II, III, or IV. Between October 2009 and January 2011, 31 children and adolescents with $\mathrm{CP}$ were identified and agreed to be contacted with respect to study participation. Of these 31 , four candidates opted not to participate, three candidates agreed to participate but later withdrew from the study, and one participant was diagnosed with an acquired brain injury and was subsequently excluded. As a result, 23 children and adolescents (17 males, 6 females; mean age $13.5 \mathrm{y}$, SD $2.6 \mathrm{y}$ ) completed the study, of which nine were classified at GMFCS level I, five level II, five level III, and four level IV. For classification of severity we used the GMFCS Expanded and Revised version (GMFCS-ER) that has excellent interrater reliability for use in adolescents (12-18 years) [14]. All parents/guardians and participants provided written consent/assent to participate in this study approved by the Faculty of Health Sciences/ Hamilton Health Sciences Research Ethics Board, Hamilton, Canada.

2.2. Assessment of Physical Activity. Habitual physical activity was objectively assessed using the ActiGraph GT1M activity monitor. This device was chosen for the purposes of this study for its ability to measure activity over a relatively prolonged period while remaining unobtrusive. The ActiGraph GT1M accelerometer weighs $27 \mathrm{~g}$ with dimensions of $3.8 \times 3.7 \times 1.8 \mathrm{~cm}$ (i.e., about the size of a matchbox) and measures and records acceleration in the vertical plane ranging from $\sim 0.05$ to $2 \mathrm{G}$ in magnitude. The acceleration is sampled and digitized by a 12-bit analog-to-digital converter at a rate of $30 \mathrm{~Hz}$. This signal is passed through a digital filter that eliminates nonhuman motion and then stored in user-defined intervals (i.e., epochs). Given the brief, intermittent, and spontaneous nature of activity reported in youth, physical activity was recorded in 3-second recording intervals or epochs [15].

2.3. Procedure. Participants were instructed to wear the accelerometer over the right hip during all waking hours for seven consecutive days, except when engaging in water activities so as not to damage the equipment. A seven-day period was selected to ensure that measured activity was representative of habitual levels of physical activity [16]. A log book was kept with the intent of tracking all times (and reasons) the device was removed and replaced. Figure 1 shows an example of a logbook and accelerometer output for a typical monitoring day for one participant. Upon completion of the seven days, log books and accelerometers were obtained from the participants and downloaded for further analysis. The research coordinator discussed any difficulties encountered and concerns that may have arisen with the participant and their parents/guardian.

2.4. Physical Activity Analysis. Accelerometer data were visually inspected to ensure that information recorded in the $\log$ book corresponded with the accelerometer output. Any activity recorded during periods of nonwear time, as indicated by the participant in the log book, was deleted. Observations of consecutive epochs of "0" counts were 


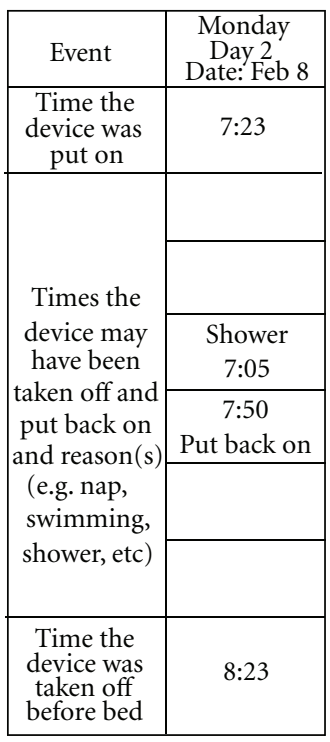

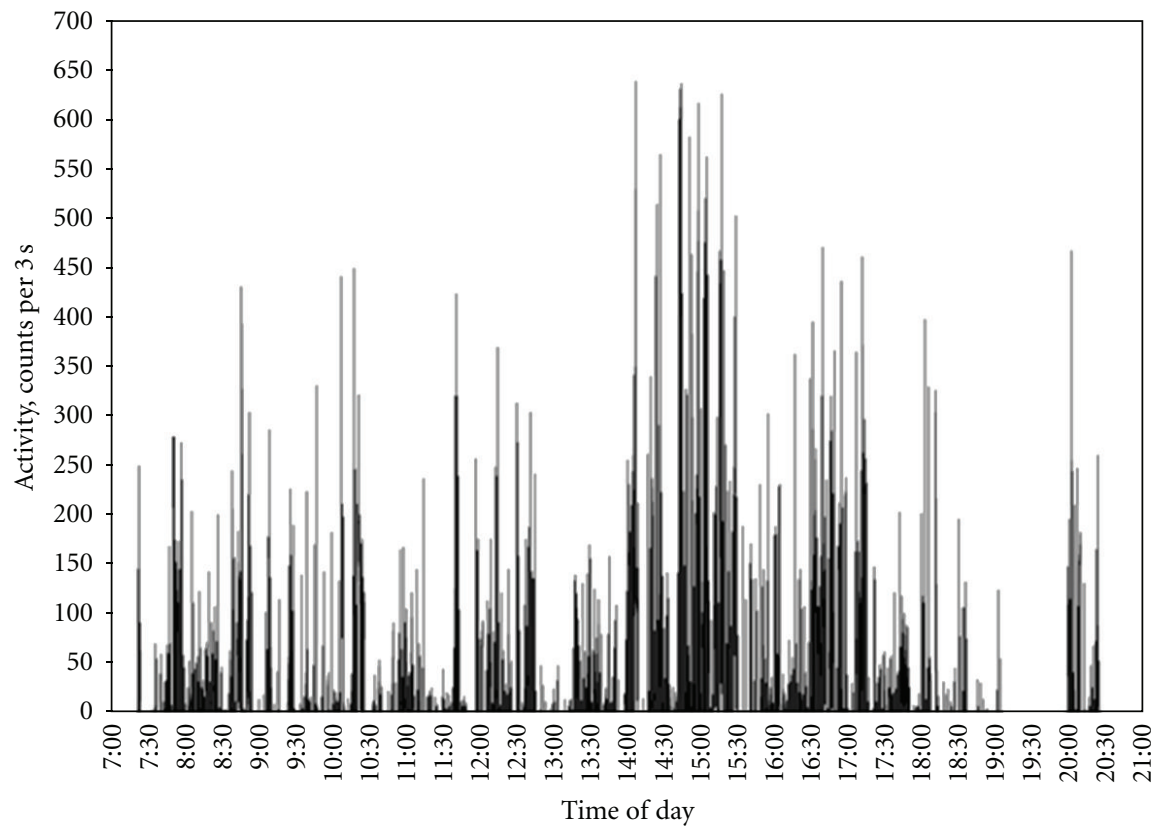

FIGURE 1: An example of a typical monitoring day for one participant with CP (female, 11 years of age, GMFCS level I). The table is a sample from the participant's log book in which they were asked to record the times the device was put on and taken off for each of the monitoring days. The figure to the right represents the accelerometer output for the corresponding day. No activity was recorded by the device from 7:05 to approximately 7:56 pm, which corresponds to the time the participants indicated they removed the device.

considered sedentary time unless otherwise stated in the log book. Only participants with $\geq 5$ hours of monitoring time on $\geq 4$ days were included in the analyses. These criteria were selected to maximize participant inclusion and were based on the minimal allowable time previously used to estimate habitual physical activity $[16,17]$. The data were then uploaded to a Microsoft Excel-based Visual Basic data reduction program to determine total monitoring time and total activity. The program also distinguished light physical activity (LPA), moderate physical activity (MPA), moderateto-vigorous activity (MVPA), and vigorous physical activity (VPA). Activity intensity was examined using the cut-points developed by Clanchy et al. and Evenson et al., which were recently validated for use in children and adolescents with CP $[12,18]$.

2.5. Descriptive Statistics. Levels of physical activity (total, LPA, MPA, VPA, and MVPA) between the four GMFCS levels were compared using one-way analysis of variance (ANOVA) in STATISTICA (StatSoft, Tulsa, Okla., USA). Analyses of covariance (ANCOVA) were also performed with chronological age as the covariate so as to account for the distribution in age among participants. Tukey's honestly significant post hoc tests were performed when necessary. Given the small number of participants in each of the GMFCS levels, Kendall's Tau was used to assess the relationship between MVPA and GMFCS level in SPSS (Version 17.0, Chicago, Ill., USA). Descriptive statistics were used to calculate the proportion of participants meeting the Canadian physical activity guideline recommendations for youth ( $\geq 60$ min MVPA per day). Statistical significance for all analyses was set at $P \leq 0.05$.

\section{Results}

3.1. Feasibility. We found that refusal to participate was most often based upon the lack of parental enthusiasm and the youth's perception that they might look "different" in various social settings. No participants reported discomfort or adverse effects of wearing the accelerometer throughout the duration of the study. One participant exposed the device to water on day 5 of wear, which resulted in highly erratic data. Therefore, that participant's data from only days 1 to 4 were included in the analyses.

3.2. Physical Activity Levels. None of the participants were excluded on the basis of failure to wear the accelerometer for the minimum required period ( $\geq 5$ hours on $\geq 4$ days). On average, the device was worn for 6 of the 7 required monitoring days, with the monitoring period ranging from 540.5 to 859.2 minutes per day (mean \pm SD: $707.7 \pm$ $81.2 \mathrm{~min}$ ). On a daily basis, our participants engaged in $($ mean \pm SD) $89.5 \pm 47.1 \mathrm{~min}$ of LPA, $17.8 \pm 16.9 \mathrm{~min}$ of MPA, $12.0 \pm 14.4 \mathrm{~min}$ of VPA, and $30.7 \pm 30.3 \mathrm{~min}$ of MVPA. To account for differences in wear time, data were also examined as minutes of activity per hour of monitoring time (Table 1). Both ANOVA and ANCOVA suggested that youth classified at GMFCS level IV presented with lower levels of LPA, MPA, and MVPA compared with level I $(P<$ 0.05). Similarly, youth at level III demonstrated lower levels 
TABLE 1: Minutes of activity per hour and per day of monitoring time.

\begin{tabular}{|c|c|c|c|c|c|c|}
\hline & \multicolumn{6}{|c|}{ GMFCS } \\
\hline & & $\begin{array}{l}\text { Level I } \\
(n=9)\end{array}$ & $\begin{array}{l}\text { Level II } \\
(n=5)\end{array}$ & $\begin{array}{c}\text { Level III } \\
(n=5)\end{array}$ & $\begin{array}{l}\text { Level IV } \\
(n=4)\end{array}$ & $\begin{array}{c}\text { Total } \\
(n=23)\end{array}$ \\
\hline \multirow[t]{2}{*}{ LPA } & $\min /$ day & $121.5(38.7)$ & $95.7(43.7)$ & $66.7(33.5)$ & $32.1(18.2)^{*}$ & $88.4(47.8)$ \\
\hline & $\mathrm{min} / \mathrm{hr}$ & $10.2(3.4)$ & $8.2(3.6)$ & $5.6(2.3)$ & $1.6(1.2)^{* \dagger}$ & $7.3(4.2)$ \\
\hline \multirow[t]{2}{*}{ MPA } & $\min /$ day & $33.0(15.6)$ & $16.4(12.5)$ & $6.0(5.7)^{*}$ & $2.7(3.7)^{*}$ & $18.2(17.1)$ \\
\hline & $\mathrm{min} / \mathrm{hr}$ & $2.7(1.2)$ & $1.4(1.0)$ & $0.5(0.4)^{*}$ & $0.2(0.3)^{*}$ & $1.5(1.4)$ \\
\hline \multirow[t]{2}{*}{ MVPA } & $\min /$ day & $56.0(28.4)$ & $26.6(24.7)$ & $9.3(10.4)^{*}$ & $5.8(8.0)^{*}$ & $30.7(30.3)$ \\
\hline & $\mathrm{min} / \mathrm{hr}$ & $4.5(2.1)$ & $2.2(2.1)$ & $1.5(1.5)^{*}$ & $0.5(0.7)^{*}$ & $2.6(2.4)$ \\
\hline
\end{tabular}

Data are presented as mean (SD). ${ }^{*}$ indicates significant difference from level I, ${ }^{\dagger}$ indicates significant difference from level II, $P<0.05$.

Minutes of activity per hour and per day of monitoring time.

LPA: light physical activity; MPA: moderate physical activity; MVPA: moderate-to-vigorous physical activity; CP: cerebral palsy; GMFCS: Gross Motor Function Classification System; min: minutes; hr: hour.

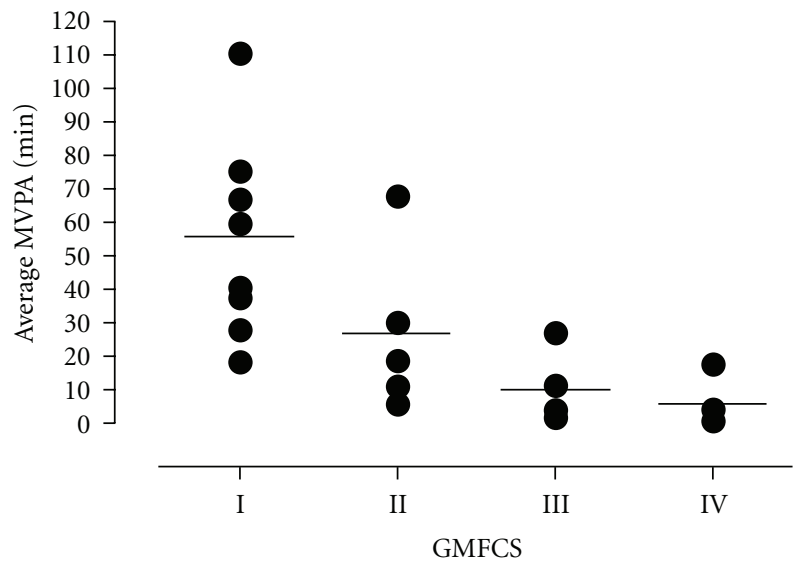

Figure 2: Moderate-to-vigorous activity time in adolescents with CP by GMFCS level. MVPA: moderate-to-vigorous physical activity; CP: cerebral palsy; GMFCS: Gross Motor Function Classification System; min: minutes.

of MPA and MVPA compared with level I. No differences were seen between levels I and II for any intensity, nor were there significant differences between levels II, III, and IV. A significant negative correlation was seen between MVPA, in both minutes/day and minutes/hour, and GMFCS levels (minutes/day: $\tau=-0.65$, minutes/hour: $\tau=-0.61, P<$ 0.001, Figure 2).

\section{Discussion}

This study aimed to assess the ability of accelerometers to measure the duration, intensity, and timing of physical activity in the home, community, and at school of ambulatory and nonambulatory adolescents with CP. The use of the accelerometer worn around the waist to measure habitual physical activity has been shown to be feasible and unobtrusive to participants, including adolescents classified as GMFCS level IV. Our results are in line with recently published data on the ActiGraph in children and teenagers up to age 16 years with CP, GMFCS level I and II $[11,12]$ and add supportive data for its use in adolescents with more functional limitations (GMFCS level III and IV) and older adolescents (up to age 20 years).

While our findings are promising, a number of limitations both inherent to the accelerometer and related to our analysis should be noted. First, our small sample size may explain the lack of significant findings when comparing activity by GMFCS level. Moreover, the activity cut-points selected were based on the work by Clanchy et al. [12], which was performed only in ambulatory youth with CP. It is unknown whether these same cut-points are applicable in nonambulatory adolescents with CP. Our assessment of activity is further complicated by the use of waist-worn accelerometer in nonambulatory adolescents. More specifically, it is impossible to determine whether the use of these waist-worn accelerometers accurately captured all activity performed by participants classified as GMFCS levels III and IV, particularly in those youth who are able to self-propel their wheelchair. Future work should employ a similar design to that utilized by Clanchy et al. [12] in which the oxygen cost of movement is assessed in conjunction with accelerometer recordings. Finally, it is important to note that the sensitivity of the accelerometer to water presents a particular challenge since aquatic exercise seems to be a preferred activity of youth with CP [19]. Despite this limitation, we believe the ability of accelerometers to measure the duration, intensity, and timing of physical activity in the home, community, and at school outweighs the limitation of missing relatively small amounts of time in a pool.

With respect to activity monitoring, of the 23 participants, none engaged in $\geq 60$ min MVPA per day recommended in the Canadian Physical Activity Guidelines for youth on a daily basis, 17 (74\%) participants did not achieve 60 min MVPA on any monitoring day, and the remaining 6 participants engaged in $\geq 60$ min MVPA on 3-5 days out of 7 . Even in participants at the most functional ability level (GMFCS level I) we observed a highly sedentary lifestyle. The minimal amount of active time found in this pilot study highlights the dire need for intervention studies.

We believe the ActiGraph accelerometer is ready to use as a measure of habitual physical activity at home, at school, and in the community in children and adolescents with CP. The recent validation of cut-points for classification of 
activity levels in this population has provided a great opportunity to use accelerometers as an outcome measure in highly needed intervention studies that emphasize the integration of physical activity and participation into daily lifestyle.

\section{Conclusion}

This study shows promise for using the Actigraph GT1M accelerometer as a feasible and meaningful measure in daily activity in adolescents with CP across GMFCS levels I-IV.

\section{Abbreviations}

$\begin{array}{ll}\text { ANOVA: } & \text { One-way analysis of variance } \\ \text { ANCOVA: } & \begin{array}{l}\text { Analyses of covariance } \\ \text { CP: }\end{array} \\ \text { GMFCS: } & \begin{array}{l}\text { Grobs Motol palsy } \\ \text { System }\end{array} \\ \text { GMFCS-E\&R: } & \text { Gross Motor Function Classification } \\ & \text { System, expanded and revised version } \\ \text { LPA: } & \text { Light physical activity } \\ \text { MPA: } & \text { Moderate physical activity } \\ \text { MVPA: } & \text { Moderate-to-vigorous physical activity } \\ \text { PA: } & \text { Physical activity } \\ \text { VPA: } & \text { Vigorous physical activity. }\end{array}$

\section{Acknowledgments}

This pilot-project is part of the Stay-FIT study and is funded by the Ontario Federation for Cerebral Palsy. The following members of the Stay-FIT study Group participated in the research program: Jan Willem Gorter, Maureen MacDonald, Robert Palisano, Peter Rosenbaum, Brian Timmons, and Virginia Wright. The authors thank Barb Galuppi, Research Coordinator of the Stay-FIT study, for her contributions to the project. Special thanks are due to the participating adolescents with $\mathrm{CP}$ and to their parents as well. A preliminary report of the findings of this study was presented by Stephen Noorduyn at the 2nd Joint Meeting of the North American Society for Pediatric Exercise Medicine (NASPEM) and the European Group of Pediatric Work Physiology in Niagara-on-the-Lake, ON, Canada in September 2010 and by Jan Willem Gorter at the annual meeting of the Ontario Association of Children's Services (OACRS) in Toronto on 7 November 2011.

\section{References}

[1] C. A. Maher, M. T. Williams, T. Olds, and A. E. Lane, "Physical and sedentary activity in adolescents with cerebral palsy," Developmental Medicine and Child Neurology, vol. 49, no. 6, pp. 450-457, 2007.

[2] K. F. Bjornson, B. Belza, D. Kartin, R. Logsdon, J. McLaughlin, and E. A. Thompson, "The relationship of physical activity to health status and quality of life in cerebral palsy," Pediatric Physical Therapy, vol. 20, no. 3, pp. 247-253, 2008.

[3] N. Owen, P. B. Sparling, G. N. Healy, D. W. Dunstan, and C. E. Matthews, "Sedentary behavior: emerging evidence for a new health risk," Mayo Clinic Proceedings, vol. 85, no. 12, pp. 11381141, 2010.
[4] E. G. Fowler, T. H. A. Kolobe, D. L. Damiano et al., "Promotion of physical fitness and prevention of secondary conditions for children with cerebral palsy: section on pediatrics research summit proceedings," Physical Therapy, vol. 87, no. 11, pp. 1495-1510, 2007.

[5] O. Verschuren, M. Ketelaar, T. Takken, P. J. M. Helders, and J. W. Gorter, "Exercise programs for children with cerebral palsy: a systematic review of the literature," American Journal of Physical Medicine and Rehabilitation, vol. 87, no. 5, pp. 404417, 2008.

[6] C. A. Maher, M. T. Williams, T. Olds, and A. E. Lane, "An internet-based physical activity intervention for adolescents with cerebral palsy: a randomized controlled trial," Developmental Medicine and Child Neurology, vol. 52, no. 5, pp. 448-455, 2010.

[7] A. A. O. M. Claassen, J. W. Gorter, D. Stewart, O. Verschuren, B. E. Galuppi, and L. J. Shimmell, "Becoming and staying physically active in adolescents with cerebral palsy: protocol of a qualitative study of facilitators and barriers to physical activity," BMC Pediatrics, vol. 11, article no. 1, 2011.

[8] World Health Organization, The International Classification of Functioning, Disability, and Health, World Health Organization, Geneva, Switzerland, 2001.

[9] A. A. Martin, L. M. Cotie, B. W. Timmons, J. W. Gorter, and M. J. MacDonald, "Arterial structure and function in ambulatory adolescents with cerebral palsy are not different from healthy controls," International Journal of Pediatrics, vol. 2012, Article ID 168209, 8 pages, 2012.

[10] R. J. Van Den Berg-Emons, A. A. L'Ortye, L. M. Buffart et al., "Validation of the physical activity scale for individuals with physical disabilities," Archives of Physical Medicine and Rehabilitation, vol. 92, no. 6, pp. 923-928, 2011.

[11] C. M. Capio, C. H. Sit, and B. Abernethy, "Physical activity measurement using MTI (actigraph) among children with cerebral palsy," Archives of Physical Medicine and Rehabilitation, vol. 91, no. 8, pp. 1283-1290, 2010.

[12] K. M. Clanchy, S. M. Tweedy, R. N. Boyd, and S. G. Trost, "Validity of accelerometry in ambulatory children and adolescents with cerebral palsy," European Journal of Applied Physiology, vol. 111, no. 12, pp. 2951-2959, 2011.

[13] K. M. Clanchy, S. M. Tweedy, and R. Boyd, "Measurement of habitual physical activity performance in adolescents with cerebral palsy: a systematic review," Developmental Medicine and Child Neurology, vol. 53, no. 6, pp. 499-505, 2011.

[14] J. W. Gorter, J. Slaman, D. Bartlett, and H. J. G. Van den BergEmons, "Reliability of the gross motor function classification system expanded and revised (GMFCS-ER) when used with adolescents and young adults with cerebral palsy," Developmental Medicine \& Child Neurology, vol. 53, supplement 5, pp. 42-43, 2011.

[15] G. Baquet, S. Berthoin, and E. Van Praagh, "Are intensified physical education sessions able to elicit heart rate at a sufficient level to promote aerobic fitness in adolescents?" Research Quarterly for Exercise and Sport, vol. 73, no. 3, pp. 282-288, 2002.

[16] S. G. Trost, R. R. Pate, P. S. Freedson, J. F. Sallis, and W. C. Taylor, "Using objective physical activity measures with youth: how many days of monitoring are needed?" Medicine and Science in Sports and Exercise, vol. 32, no. 2, pp. 426-431, 2000.

[17] K. A. Pfeiffer, M. Dowda, K. L. McIver, and R. R. Pate, "Factors related to objectively measured physical activity in preschool children," Pediatric Exercise Science, vol. 21, no. 2, pp. 196-208, 2009. 
[18] K. R. Evenson, D. J. Catellier, K. Gill, K. S. Ondrak, and R. G. McMurray, "Calibration of two objective measures of physical activity for children," Journal of Sports Sciences, vol. 26, no. 14, pp. 1557-1565, 2008.

[19] J. W. Gorter and S. J. Currie, "Aquatic exercise programs for children and adolescents with Cerebral Palsy: what do we know and where do we go?" International Journal of Pediatrics, vol. 2011, Article ID 712165, 7 pages, 2011. 


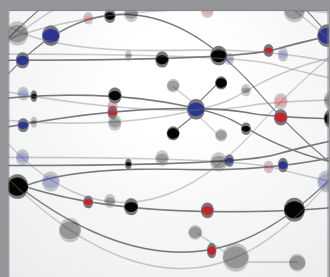

The Scientific World Journal
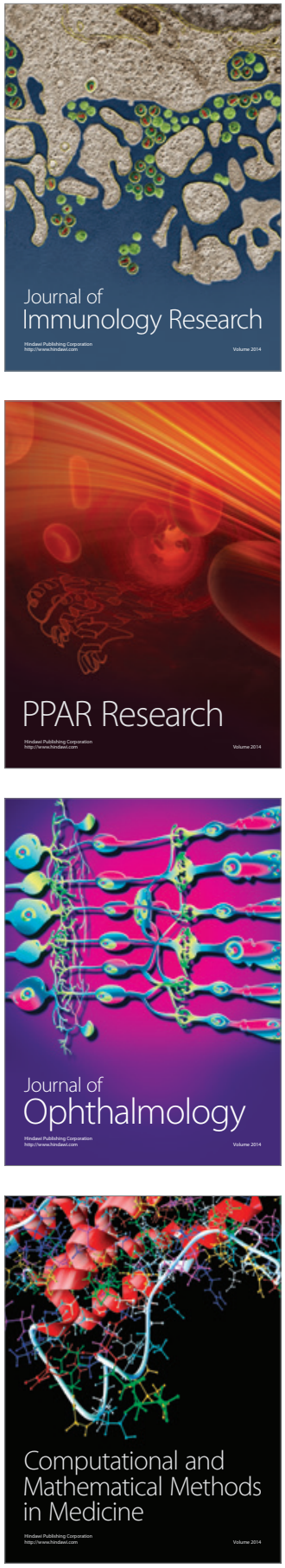

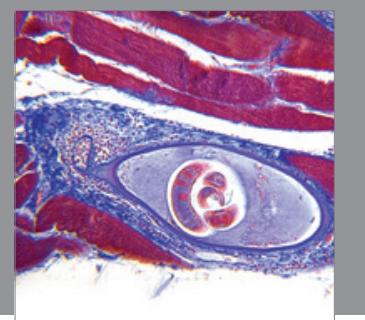

Gastroenterology

Research and Practice
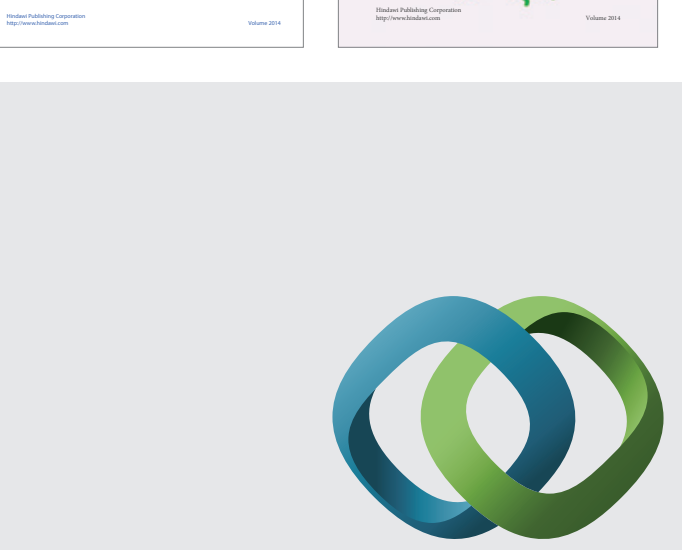

\section{Hindawi}

Submit your manuscripts at

http://www.hindawi.com
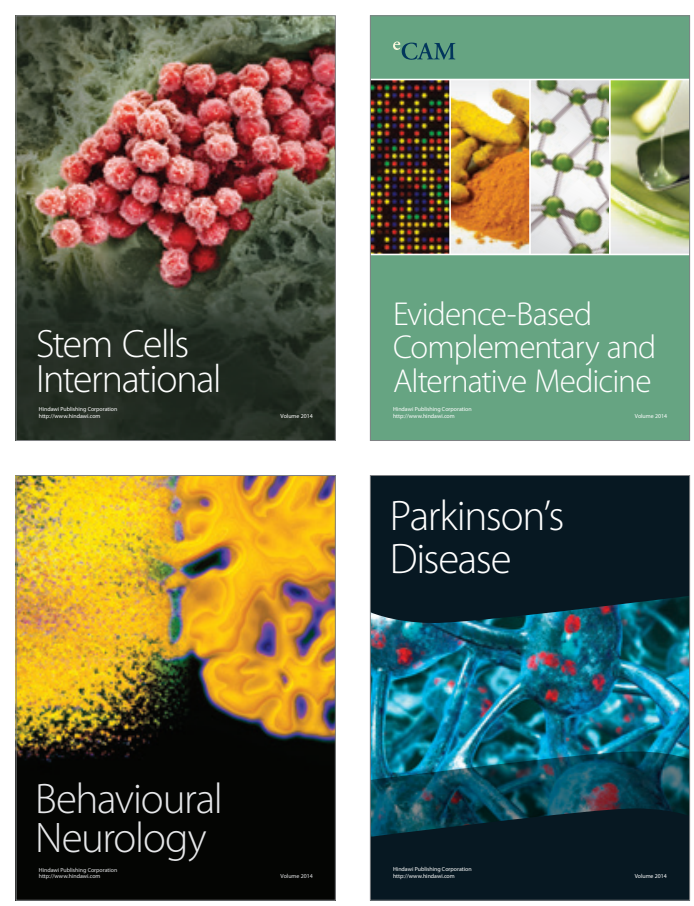

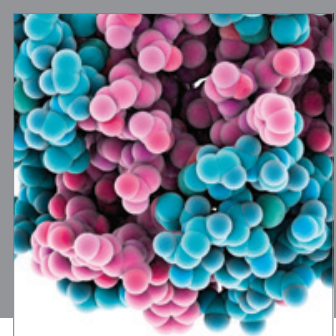

Journal of
Diabetes Research

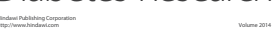

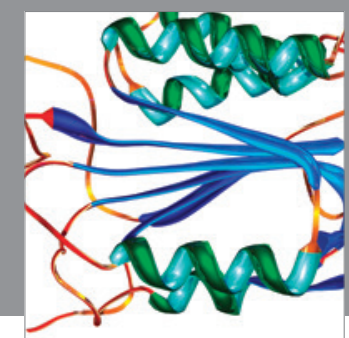

Disease Markers
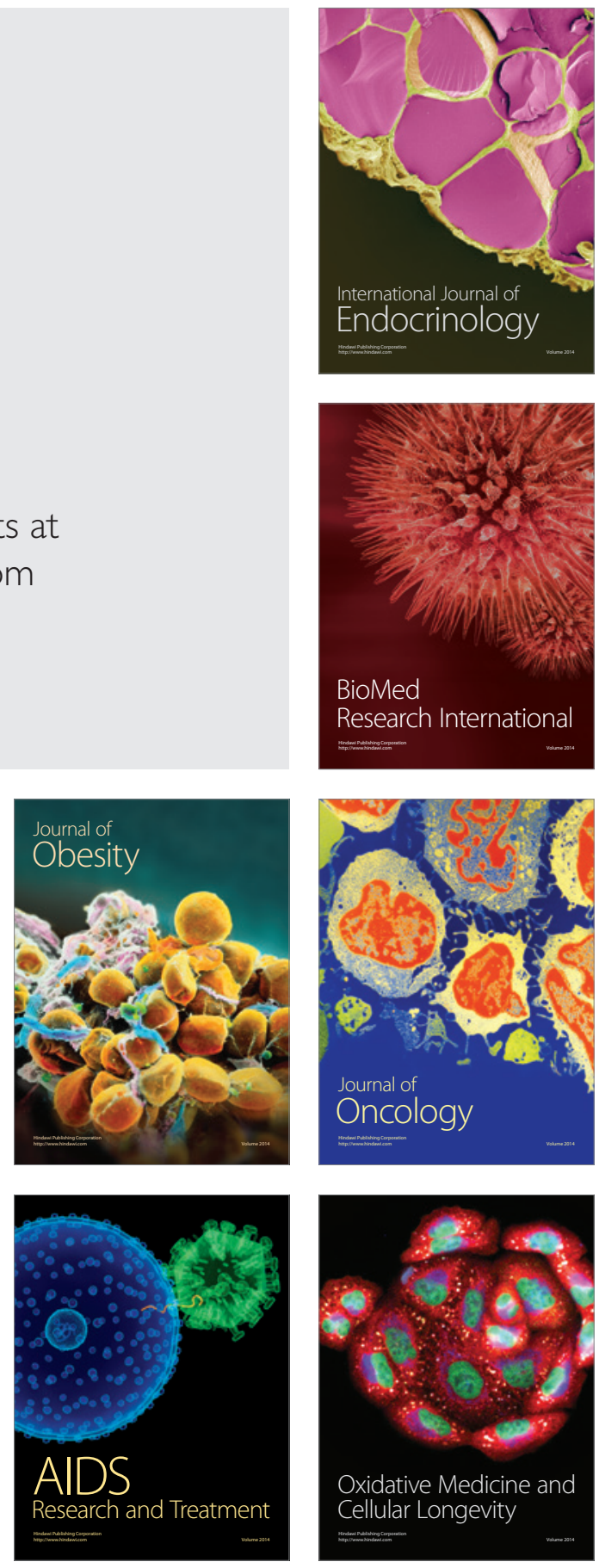